\title{
Surface acoustic waves in finite slabs of three-dimensional phononic crystals
}

\author{
R. Sainidou, * B. Djafari-Rouhani, and J. O. Vasseur \\ Institut d'Electronique, de Microélectronique, et de Nanotechnologie, UMR CNRS 8520, Avenue Poincaré, Boîte Postale 60069, \\ 59652 Villeneuve d'Ascq Cedex, France
}

(Received 1 October 2007; published 18 March 2008)

\begin{abstract}
We study theoretically, by means of layer-multiple-scattering techniques, the propagation of elastic waves through finite slabs of phononic crystals consisting of metallic spheres in a polyester matrix, embedded in air. In particular, we focus on the study of modes localized on the surfaces of the structure. Their origin and behavior, as well as the physical parameters that influence and determine their appearance, are investigated in detail. Our results reveal the existence of absolute phononic frequency gaps in these finite structures, and point out the possibility, under an appropriate choice of the parameters, of tunable regions of frequency free of propagating and/or surface-localized modes.
\end{abstract}

DOI: 10.1103/PhysRevB.77.094304

PACS number(s): 63.22. $-\mathrm{m}$, 43.20. $+\mathrm{g}$, 68.35.Ja, 68.35.Iv

\section{INTRODUCTION}

In recent years, the research related to phononic crystals, composite structures consisting of periodically arranged macroscopic inhomogeneities of a given material (scatterers) in an otherwise homogeneous matrix of different elastic coefficients (mass density $\rho$ and elastic constants $c_{i j}$ ), has increased rapidly. ${ }^{1}$ The simplest case is that of cylindrical or spherical scatterers, and in such a case we name the corresponding composite structures two-dimensional (2D) or three-dimensional (3D) phononic crystals, respectively. The interest in these materials was, especially at the beginning, stimulated by the investigation of absolute frequency gaps in these structures, i.e., regions of frequency where no elastic waves can propagate inside the crystal, independently of their polarization and angle of direction of propagation. Nowadays, the research on phononic crystals increasingly focuses on nonperiodic structures and related phenomena such as disorder effects, sound focusing, waveguiding, etc. In this framework, the study of surface elastic waves associated with the surface of a semi-infinite phononic crystal, or of the plate modes in a finite-thickness phononic crystal, has attracted a lot of attention in recent years, in the domain of basic research, ${ }^{2-17}$ but also because of its promise in technological applications, mostly in telecommunications, ${ }^{18,19}$ in microelectromechanical systems, ${ }^{20}$ etc. On the other hand the investigation of gaps in the frequency spectra of the abovementioned finite structures is important again, as it is the base for the development of defect or guided modes in a region free of modes. All the above facts clearly point out the need of a thorough understanding of the behavior of surface and slab modes from a fundamental point of view. The relevant literature is so far almost exclusively focused on systems of 2D phononic crystals, which have been extensively studied theoretically ${ }^{2-10}$ and experimentally. ${ }^{11-16}$ In these studies cases of both semi-infinite $e^{2-6,14-16}$ and finite ${ }^{7-13}$ slabs are considered. The corresponding 3D systems remain from this point of view practically unexplored, apart from one exception, ${ }^{17}$ as far as we know.

In the theoretical study of propagation of elastic waves through a finite phononic-crystal slab, one assumes in the simplest case that the slab is embedded in a material different from the one surrounding the scatterers (spheres or cylinders), and, usually, in an actual experiment in a fluid (water or air) or even in vacuum. For a slab finite along the $z$ direction (the direction normal to its surface), the description of the modes of this system can be realized through its dispersion diagram $\left(\omega, \mathbf{k}_{\|}\right)$, where $\mathbf{k}_{\|}$is the component of the wave vector of the elastic wave reduced in the corresponding surface Brillouin zone (SBZ) of the 2D periodic lattice. Here, we are interested in the study of surface modes of a slab made of a 3D phononic crystal; therefore we will consider it sufficiently thick. In such a case one in general expects that most of the modes of the slab reproduce the bulk bands of the phononic crystal, while some (a few) of them will appear in the frequency range of the absolute band gap of the phononic crystal, having their origin in the two end surfaces of the slab. They clearly represent the surface waves of a semi-infinite crystal. In this respect, we take advantage of the existence of an absolute band gap in the band structure of a 3D phononic crystal, which will be translated as a gap in the dispersion diagram of a slab or even of a semi-infinite crystal. This is in contrast to the case of a 2D phononic crystal. Indeed, for an infinite 2D crystal, the absolute band gap exists only for in-plane propagation and disappears when propagation along the $z$ direction (parallel to the cylinders) is taken into account. On the other hand, it should be remembered that a finite-thickness slab of a 2D phononic crystal (where the cylinders are placed with their axes of symmetry normal to the surface and their lengths equal the thickness of the slab) can still exhibit an absolute band gap ${ }^{7-9}$ provided its thickness is chosen to be of the order of the lattice period. Therefore, this band gap disappears if the slab is thick enough to mimic the situation of a semi-infinite crystal, whereas for a thin plate it is not appropriate in general to make a distinction between bulk and surface modes. We note here that in the discussion of the modes of a (sufficiently thick) slab we adopt the term "propagating modes" for those modes that coincide in frequency with the bulk bands of the infinite crystal and whose $z$ spatial behavior is oscillatory. Correspondingly, we will use the term "surface-localized modes" for those modes appearing within the band gaps of the infinite crystal and having an evanescent behavior along the $z$ direction. 
Slabs made of 3D phononic crystals offer more possibilities for the tailoring of the surface-mode properties, since their geometrical configuration allows the use of more degrees of freedom. We assume here for simplicity that the surfaces are parallel to a specific crystallographic direction of the $3 \mathrm{D}$ crystal. The $3 \mathrm{D}$ finite slab can therefore be viewed as a repetition of $N_{L}$ parallel identical planes of spheres of a given crystallographic direction (layers), with a distance $d$ separating them. Each of the two end surfaces is located at a distance $d_{s}=d / 2$ from the center of each surface plane of spheres, but of course along the $z$ direction more complicated structures can also be constructed. The slab can be a sequence of different layers, where in this most general case we adopt the term "layer" for any of the following: a plane of spheres, a homogeneous plate, or a single interface. In this manner, one can modify the surface layers, and consequently the properties of the surface modes of this slab (by changing the radius of the spheres of the surface layer, and/or the distance between the end surface and the plane of the spheres, or by adding extra surface homogeneous layers) without destroying the crystal structure in the internal layers and, therefore, the possible useful properties and features that result from it.

In the present paper we are concerned with the study of surface-localized modes in finite, but relatively thick, slabs of 3D solid phononic crystals, embedded in air. We investigate in detail the evolution of these modes by changing the surface properties of these slabs, and we demonstrate the possibility of tuning their position inside the absolute gap by means of these surface characteristics. We also study the behavior of these modes below the first passbands of the corresponding infinite system, offering a simple way to create tunable regions of frequency free of propagating and/or surface modes.

In Sec. II we briefly give the outline of the method used for our calculations, and in Sec. III we present the results obtained by this method and the relevant discussion. Finally, we conclude the paper in Sec. IV.

\section{METHOD OF CALCULATION}

For the study of propagation of elastic waves in finite slabs of 3D phononic crystals, and especially in slabs of layered form along the $z$ direction, normal to their surfaces, the layer-multiple-scattering method as developed for phononic crystals ${ }^{21-23}$ is an appropriately chosen tool as it does not require periodicity in the $z$ direction. The method can treat efficiently, in addition to an infinite phononic crystal, also a slab of the crystal of finite thickness which may be, in the general case, a succession of different layers, which can be single interfaces, homogeneous slabs, or planes of spheres arranged with a given 2D periodicity on each plane. The only requirement of this method is that the $2 \mathrm{D}$ periodicity must always be the same among the different layers. This implies that $\mathbf{k}_{\|}$, the component of the wave vector of the elastic wave reduced in the corresponding SBZ of the $2 \mathrm{D}$ periodic lattice, must be a conserved quantity. For each layer, the method calculates the transmission and reflection matrices, $\mathbf{Q}^{\mathbf{I}}$ and $\mathbf{Q}^{\text {III }}$, respectively, for a plane wave incident on the layer with given frequency and $\mathbf{k}_{\|}$from the left (i.e., with $k_{z}>0$ ), as well as the corresponding matrices $\mathbf{Q}^{\mathbf{I V}}$ and $\mathbf{Q}^{\mathbf{I I}}$ for incidence from the right (i.e., with $k_{z}<0$ ). Explicit expressions for these $\mathbf{Q}$ matrices can be found elsewhere. ${ }^{22}$ If one is interested in studying the propagation of elastic waves of frequency $\omega$ incident from the one side (e.g., from the left) of the finite slab with a given $\mathbf{k}_{\|}$, then the layer-multiple-scattering formalism provides us with a set of transmission and reflection matrices, for the whole finite structure. These matrices are calculated from those of the constituent layers, and through them the transmittance and reflectance of this slab for a wave of given $\omega, \mathbf{k}_{\|}$, and polarization, as well as the corresponding density of states of the elastic field, are obtained. ${ }^{22,23}$

Moreover, the method can also be applied to calculate the dispersion diagrams $\left(\omega, \mathbf{k}_{\|}\right)$for a finite slab, i.e., to determine the eigenfrequencies of the bound and virtual bound states of such a structure. ${ }^{17}$ In this case, one needs to consider an appropriately chosen internal point (or, more correctly, a plane parallel to the surfaces of the slab) inside the slab, which separates the slab into a left and a right part with corresponding reflection matrices $\mathbf{Q}_{L}^{\mathrm{II}}$ and $\mathbf{Q}_{R}^{\mathrm{III}}$. The condition of bound states is then given by the equation

$$
\operatorname{det}\left(\mathbf{I}-\mathbf{Q}_{L}^{\text {II }} \mathbf{Q}_{R}^{\text {III }}\right)=0 \text {, }
$$

which results from the requirement of the existence of a wave field localized within the slab. All the quantities entering into Eq. (1) are functions of $\omega$ and $\mathbf{k}_{\|}$. For a given value of the latter, one solves the above secular equation to obtain the real- or imaginary-frequency solutions that correspond to bound or virtual bound states (leaky modes) of the system under consideration.

\section{RESULTS AND DISCUSSION}

To begin with, we consider a fcc crystal, of lattice constant $a_{0} \sqrt{2}\left(a_{0}\right.$ is the first-neighbor distance), consisting of steel spheres (mass density $\rho_{\mathrm{s}}=7800 \mathrm{~kg} / \mathrm{m}^{3}$, longitudinal and transverse velocities $c_{l \mathrm{~s}}=5940 \mathrm{~m} / \mathrm{s}$ and $c_{t \mathrm{~s}}=3200 \mathrm{~m} / \mathrm{s}$ ) in polyester (mass density $\rho=1220 \mathrm{~kg} / \mathrm{m}^{3}$, longitudinal and transverse velocities $c_{l}=2490 \mathrm{~m} / \mathrm{s}$ and $c_{t}=1180 \mathrm{~m} / \mathrm{s}$ ). The spheres have a radius $S=0.325 a_{0}$, leading to a value $f$ $=(4 \pi \sqrt{2} / 3)\left(S / a_{0}\right)^{3}=0.20$ for the volume filling fraction of the crystal. This system, in its infinite form, is known to present large absolute gaps, originating from the interaction of the dipole resonances of the scatterers with the effective medium bands which describe the propagation in a homogeneous medium. ${ }^{24}$ This type of gap, the so-called hybridization gaps, in opposition to the Bragg gap, can be present even with only one plane of spheres. In the case considered here this gap extends from $\omega a_{0} / c_{\text {air }}=16.72$ to 23.63. In Fig. 1 (a) we show the projection of the frequency band structure of the acoustic field in this crystal on the SBZ of its (001) surface, which we calculated using the computer program of Ref. 22. The shaded regions extend over the frequency bands of the acoustic field: at any one frequency within the shaded region, for a given $\mathbf{k}_{\|}$[the component of the reduced wave vector within the SBZ of the (001) surface], there exists at least one propagating mode [the corresponding $k_{z}(\omega)$ is real] 

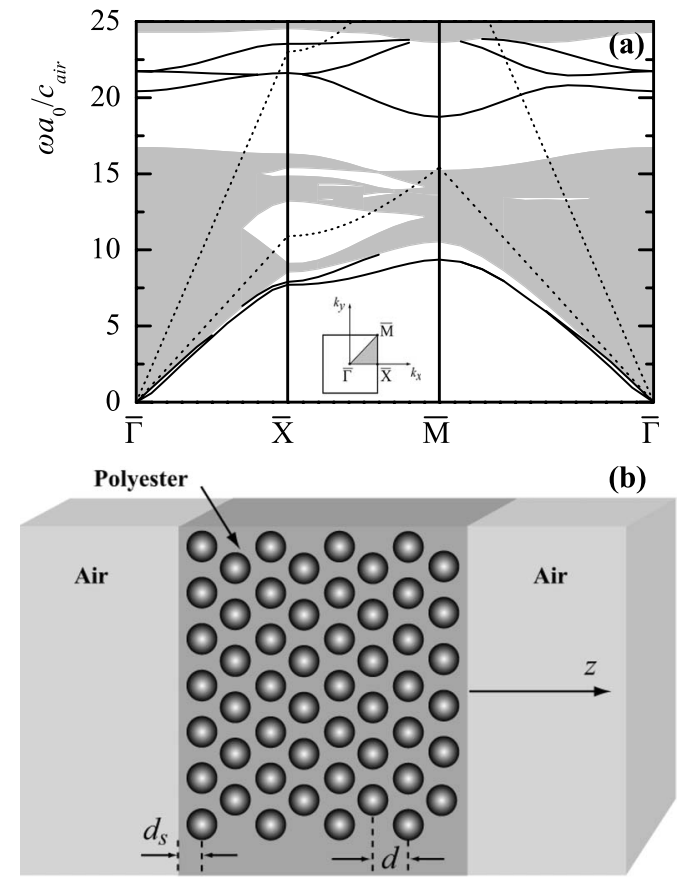

FIG. 1. (a) Projection of the phononic band structure of a fcc crystal of steel spheres (radius $S=0.325 a_{0}$ ) in polyester along the high-symmetry lines of the SBZ of its (001) surface (shown in the inset). Propagating modes, shaded regions; gaps, blank regions. Solid lines: additional modes of the corresponding finite slab consisting of eight (001) layers of spheres, embedded in air [see (b)]. Dotted lines: lines of propagation of longitudinal and transverse waves in infinite polyester (higher and lower branch, respectively). (b) Slab finite along the $z$ direction, as described in the text. $d$ and $d_{s}$ denote, respectively, the distance between two successive planes of spheres and between the air-polyester interface and the center of the surface plane of spheres.

of the acoustic field in the infinite crystal. The blank areas correspond to frequency gaps [all $k_{z}(\omega)$ are complex]. Over the frequency region of the absolute gap, from $\omega a_{0} / c_{\text {air }}$ $=16.72$ to 23.63 , there are no propagating modes of the acoustic field in the infinite crystal. We verified that this is indeed an absolute gap by calculating the band structure at a sufficient number of $\mathbf{k}_{\|}$points within the SBZ.

We next consider the corresponding finite slab of this crystal, constructed as a sequence of $N_{L}$ successive fcc (001) planes of spheres (layers), with a distance $d=a_{0} \sqrt{2} / 2$ between them, and for the present study we assume it sufficiently thick: we consider $N_{L}=8$. The whole structure is embedded in air (mass density $\rho_{\text {air }}=1.23 \mathrm{~kg} / \mathrm{m}^{3}$, sound velocity $c_{\text {air }}=340 \mathrm{~m} / \mathrm{s}$ ) with the two end surfaces at a distance $d_{s}$ from the center of the surface sphere planes, as shown in Fig. 1(b). Of course $d_{s}$ can vary, following the inequality $d_{s} \geq S$ (the spheres cannot be cut from the air-polyester interface). We assume $d_{s}=d / 2$, unless otherwise stated. For this finite system as described above, we find the modes of the slab propagating along the $z$ direction which reproduce in a discrete way the regions of elastic states of the infinite crystal [shaded regions in Fig. 1(a)]: each band of the infinite system corresponds in the finite-system case to a discrete number of points directly related to the number of layers of spheres along the $z$ direction. For sufficiently thick slabs (which is the case here), the frequency limits of these two representations are practically the same; therefore, in what follows we represent the propagating modes of the finite system by the continuous (shaded) regions of the corresponding infinite system. We note here that the use of the term "propagating modes" refers to the $z$ direction: these modes of a finite slab have an oscillatory behavior inside the slab region, along this direction. Apart from these propagating modes of the slab, additional modes originating from the presence of the two end surfaces of the finite system (air-polyester interfaces) appear, shown by the solid lines in Fig. 1(a). In the region of the absolute gap they extend from $\omega a_{0} / c_{\text {air }}=18.74$ to 23.63 and of course they continue upward, penetrating within the region of propagating modes, but we are not interested in those regions. We focus our study only on such modes lying within the frequency-gap regions of the corresponding infinite system. In the low-frequency region (i.e., in the first frequency gap) they follow in general the shape of the first passband, and are located always a little bit lower, at a small distance from it. For slabs sufficiently thick, which is the case here, these modes are doubly degenerate, each of them associated with each one of the two surfaces (apart from the low-frequency region, i.e., for $\omega a_{0} / c_{\text {air }} \lesssim 5$, where a split is observed because of the strong repulsion of the two corresponding modes). Strictly speaking, the above-mentioned modes are not surface modes, in the sense that they do not decay exponentially on both sides of the air-polyester interfaces: they propagate along the $z$ direction in air regions, as they are higher in frequency than the sound propagation line in air $\left(\omega a_{0} / c_{\text {air }}=\left|\mathbf{k}_{\|}\right| a_{0}\right)$. However, one expects that they will be strongly localized in the surface regions, i.e., between the air-polyester interface and the first sphere planes: they attenuate in the interior of the thick phononic-crystal slab, in the gap-frequency regions of the corresponding infinite system. We shall call them "surface-localized modes" to distinguish them from the above-mentioned "propagating modes."

The orientation of the surfaces of the slab does not leave unaffected the form of these modes in the corresponding dispersion curves. Keeping the same infinite system (same crystal structure, same materials and filling fraction), we can choose the surfaces of the slab parallel to the (111) planes. The distance between successive (111) planes is now $d$ $=a_{0} \sqrt{6} / 3$ and again we assume $d_{s}=d / 2=a_{0} \sqrt{6} / 6=0.408 a_{0}$, unless otherwise stated. Obviously, the position in the frequency of the absolute gap of the infinite structure remains the same, independently of the crystallographic orientation of the surfaces, as can be seen in Fig. 2. In other words, the propagating modes in the finite structure pertain to the same frequency band regions, although the detailed form of the projection of the band structure in the corresponding SBZ associated with the (111) surface changes. However, this is not the case for the surface-localized modes lying inside the gaps. Compared to the previous case [see Fig. 1(a)] they are in general less extended in frequency: from $\omega a_{0} / c_{\text {air }}=22.68$ to 23.63 in the region of the absolute gap, and almost dispersionless along the $\overline{K M}$ direction in the first phononic gap. In what follows, we shall consider this system as the base for our study and we will refer to it as the reference system. 


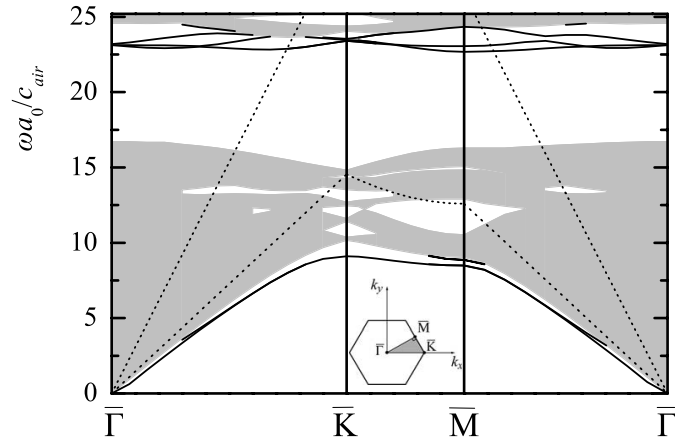

FIG. 2. Dispersion curves for a finite slab consisting of eight fcc (111) planes of steel spheres of radius $S=0.325 a_{0}$ in a polyester host, embedded in air, along the high-symmetry lines of the SBZ of its (111) surface (shown in the inset). Propagating modes, shaded regions; gaps, blank regions. Solid lines: surface-localized modes. Dotted lines: lines of propagation of longitudinal and transverse waves in infinite polyester (higher and lower branch, respectively).

\section{A. The region of absolute frequency gap}

One expects that for a given crystal structure and surface orientation these modes appearing within the gaps will also be sensitive to appropriate modifications of the slab surfaces. The latter can be realized through a variety of methods in finite 3D phononic-crystal slabs. In such a case one takes advantage of the ability of $3 \mathrm{D}$ structures to generate more complicated layered structures based on the same initial 3D crystal. Using the periodicity of the internal layers to create regions of frequency where the waves attenuate in any direction of propagation inside them, we can at the same time tune the position in frequency inside the gaps of the internal crystal structure of the modes originating from the surface layers by modifying these surface layers. In Fig. 3 we demonstrate such an effect by changing the distance $d_{s}$ between each of the two end surfaces of the finite slab and the center of the surface planes of spheres. When the two surfaces are placed just at the position where the surface layers of spheres terminate $\left(d_{s}=0.325 a_{0}=S\right)$, the surface-localized modes are completely suppressed at the top of the absolute gap, and the inverse happens as we progressively increase $d_{s}$ : they move downward, becoming more dispersive, and destroy completely the full region of this gap.

Similar behavior can be obtained through modifications of the surface planes of spheres (e.g., by changing the material and/or the size of the spheres), although the detailed picture will be different from those presented in Fig. 3. As an example, we consider a slab of the phononic crystal consisting of ten planes of spheres, two of which, the external planes (so-called surface layers), may be different from the other eight. They all have, of course, the same 2D periodicity, but their spheres have a smaller or larger radius $S_{S}$ compared to those of the spheres of the internal planes, $S$. Our results are summarized in the six diagrams of Fig. 4. The first four, Figs. 4(a)-4(d), correspond to cases with $S_{S}<S$, the fifth gives the reference system $\left(S_{S}=S\right)$, as presented in Fig. 2, and the last one is for $S_{s}>S$. The general trend is similar to those of the previous case (Fig. 3), with the only difference that now, when $S_{s}$ is decreased progressively, new modes appear also at the bottom of the absolute gap.

In the two cases presented here (Figs. 3 and 4) the picture obtained for the surface-localized modes is not exactly the same, because different mechanisms are responsible for their behavior. The latter can be qualitatively described by using some simple arguments. It is well known ${ }^{25}$ that a homogeneous slab of thickness $\tilde{d}$ sandwiched on both sides by semiinfinite homogeneous media presents waveguide modes (modes localized in its interior and propagating along it, parallel to the surfaces) determined by the thickness $\widetilde{d}$ of the slab. Its dispersion plot given in dimensionless units $\left(\omega \tilde{d} / c,\left|\mathbf{k}_{\|}\right| \widetilde{d}\right)$, where $\mathbf{k}_{\|}$is the component of the wave vector parallel to the surfaces and $c$ one of the velocities of the materials of the structure, is independent of the choice of $\tilde{d}$. In the first case (Fig. 3), progressive addition of a thin homogeneous polyester layer corresponds, roughly speaking, to an effective surface homogeneous layer of increasing thickness $\tilde{d}$, sandwiched between air at its left and the phononiccrystal slab at its right. By increasing the thickness $\tilde{d}$ of such a layer (if one follows the simplified image of a homoge-
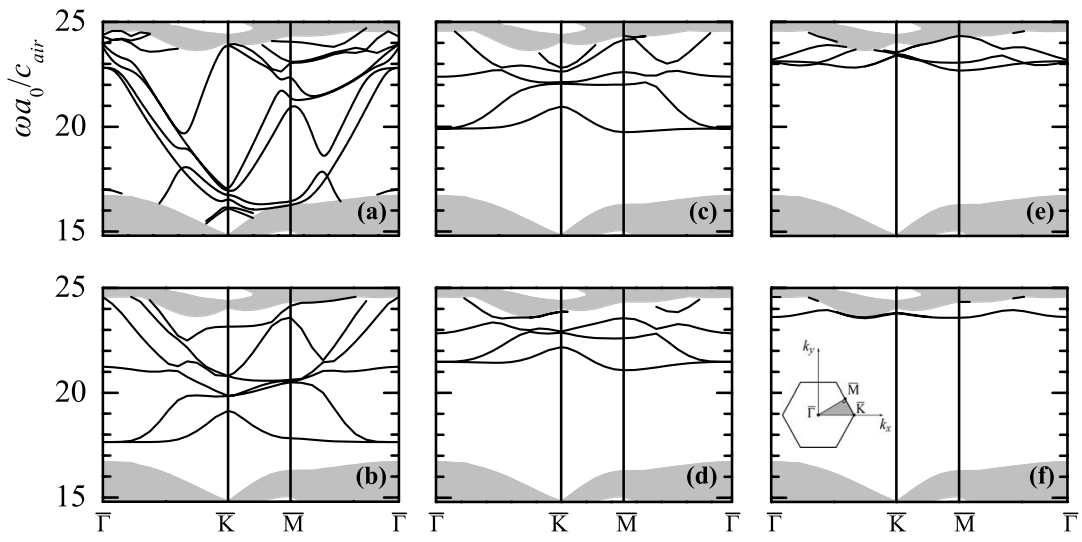

FIG. 3. Dispersion curves in the region of the absolute frequency gap of the infinite fcc crystal, for a slab consisting of eight fcc (111) layers of steel spheres of radius $S=0.325 a_{0}$ in a polyester matrix, embedded in air. The two surfaces are placed at a distance $d_{s}$ from the center of the surface planes of spheres $\left[d_{s}=\right.$ (a) $0.908 a_{0}$, (b) $0.608 a_{0}$, (c) $0.508 a_{0}$, (d) $0.458 a_{0}$, (e) $0.408 a_{0}=d / 2$ (reference system), and (f) $\left.0.325 a_{0}=S\right]$. 

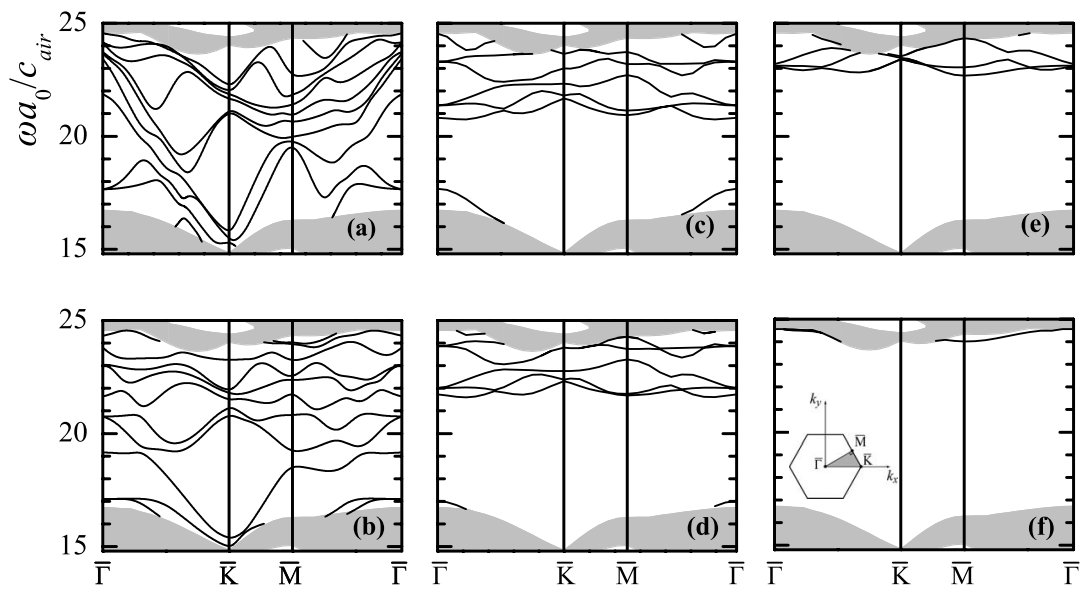

FIG. 4. Dispersion curves in the region of the absolute frequency gap of the infinite fcc crystal, for a slab consisting of ten fcc (111) layers of steel spheres in a polyester matrix, embedded in air. The two surface layers consist of spheres of radius $S_{s}\left[S_{s}=(\mathrm{a}) 0.10 a_{0}\right.$, (b) $0.20 a_{0}$, (c) $0.28 a_{0}$, (d) $0.30 a_{0}$, (e) $0.325 a_{0}=S$ (reference system), and (f) $0.35 a_{0}$, while the eight remaining internal layers are of radius $S$ $=0.325 a_{0}$.

neous slab placed on the surfaces) the waveguide modes of the slab have to move to lower frequencies $\omega$, so that $\omega_{R} \tilde{d}$ $=$ const, $\omega_{R}$ being the resonance frequency of the waveguide mode of given $\mathbf{k}_{\|}$. This explains why an increasing number of localized modes come downward from the top region of the absolute gap of the infinite phononic crystal, and fill it gradually, when one increases $d_{s}$. Of course the real picture is more complicated, since the surface layer contains an array of spheres and its behavior cannot be quantitatively described by an effective homogeneous slab of frequencyindependent elastic parameters. It is expected that the form of the dispersion plot of the modes of such a layer [an example for a slab containing a monolayer of spheres is given in Fig. 5(a)] will be the result of the folding of the dispersion lines due to the 2D periodicity and of the interaction of the homogeneous-slab modes with those originating from the individual scatterers, leading to a possible hybridization gap. The latter will be valid only if the scatterers (spheres) can support eigenmodes, and the case we study here (metallic spheres in a polyester host) is such a case. The whole picture obtained is an interplay between the homogeneous slab and the influence of the presence of the spheres, which becomes dominant for small $d_{s}$ values, thus creating the hybridization gap. When $d_{s}$ increases, the upper limit of this gap moves downward, and finally penetrates within the propagatingmode region located below the absolute gap of the phononic crystal. Its lower limit cannot be seen: in all cases presented here, it is "buried" within this region.

In the second case (Fig. 4), again the idea of an effective surface layer of appropriate thickness whose modes are superposed on those of the infinite system can be used to qualitatively describe the obtained picture; however, the surfacelayer radius $S_{s}$ dominates in the determination of the limits of the hybridization gap, which differ from those of the reference system $\left(S_{s}=S\right)$ when $S_{s} \neq S$. For a system of steel scatterers in a polyester host arranged in a fcc structure, such a gap begins to open up for $S_{s} \approx 0.24 a_{0}$, and for the range of sphere radii $S_{s}$ shown in Fig. 4 it develops almost symmetrically around its center, located at about $\omega a_{0} / c_{\text {air }} \approx 19.5$, as we increase $S_{s}{ }^{24}$ Consequently, the top and bottom of the gap associated with the surface planes of spheres of radius $S_{s}$ are pushed simultaneously upward and downward by increasing $S_{s}$, while its center frequency remains almost constant at $\omega a_{0} / c_{\text {air }} \approx 19.5$.

Independently of the mechanism that determines the detailed behavior of the modes lying within the absolute-gap region of the corresponding infinite system, these modes can be tuned in frequency as has been shown in Figs. 3 and 4 through an appropriate choice of the geometrical parameters of the surface layers $\left(d_{s}\right.$ and $S_{s}$, respectively) of the considered finite structure.

\section{B. Physical origin of the modes}

The results presented so far imply that these modes appearing within the gap regions of the corresponding infinite crystal are surface-localized modes. For a deeper understanding of their origin one needs to make a more detailed study, and to begin with we calculate the dispersion diagram of a slab consisting of only one (111) plane of spheres of radius $S=0.325 a_{0}$ embedded in polyester. The slab is of thickness $d=a_{0} \sqrt{6} / 3$ and embedded in air. The corresponding dispersion diagram is shown in Fig. 5(a). One can clearly see the three branches at the long-wavelength limit $(\omega \rightarrow 0)$, which of course are deformed with respect to a purely homogeneous slab because of the folding at the $\bar{K}$ and $\bar{M}$ points due to the periodicity occurring at about $\omega a_{0} / c_{\text {air }} \approx 11.5$ and because of the opening of a large hybridization gap extending from $\omega a_{0} / c_{\text {air }}=16.10$ to 21.52 (six bands are observed below the gap). This (absolute) gap results from the interaction of the Lamb modes of the effectively homogeneous slab with those originating from the strong interaction of dipole resonances of metallic spheres lying on the plane. If successive fcc (111) layers of spheres are added one by one to create a thicker slab, these modes are progressively suppressed toward higher frequencies. For thin slabs the two surfacelocalized modes appearing in pairs, each of them associated with each one of the two surfaces, interact strongly with each 

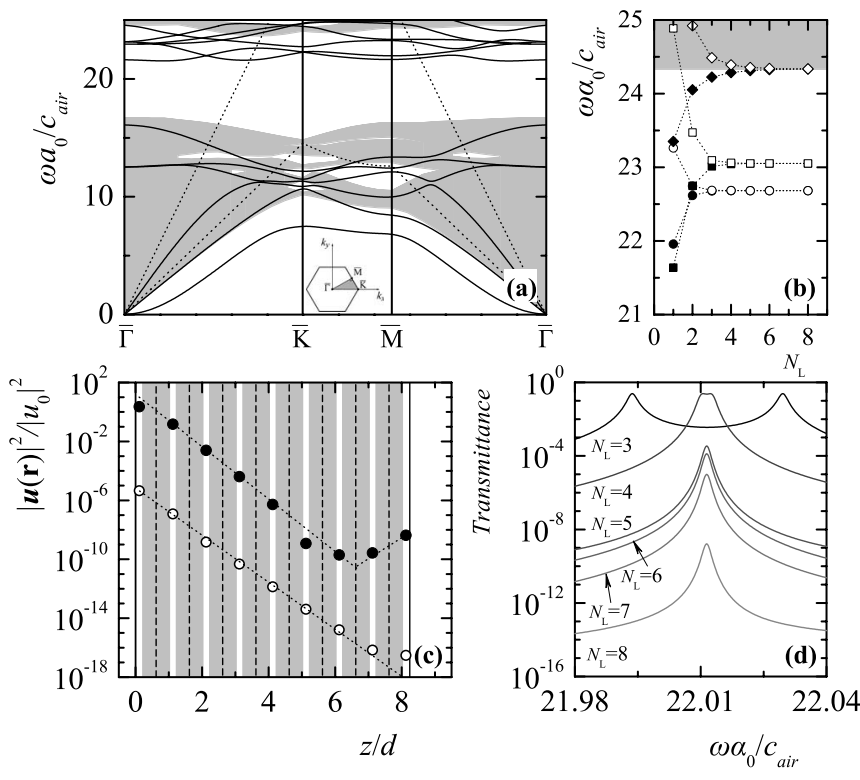

FIG. 5. (a) Dispersion curves for a slab consisting of one fcc (111) plane of steel spheres of radius $S=0.325 a_{0}$ in a polyester host, embedded in air. The propagation modes of the corresponding infinite crystal (shaded region) are also shown for comparison. Dotted lines: propagation lines of elastic waves in polyester. (b) The change in the position of the frequencies of the surface-localized modes at the $\bar{M}$ point, as a function of the thickness of the slab $\left[N_{L}\right.$ is the number of (111) layers]. (c) Normalized field-intensity distribution at the center of interlayer regions for the case of the slab described in Fig. 3(c) $\left(d_{s}=0.508 a_{0}\right)$ when a longitudinal wave of frequency $\omega a_{0} / c_{\text {air }}=22.011$ (on resonance, filled symbols) and $\omega a_{0} / c_{\text {air }}=21.00$ (off resonance, open symbols) is incident on it with wave vector $\mathbf{k}_{\|}^{\bar{M}}$. The gray strips denote the space occupied by the layers of spheres whose centers lie on the vertical dashed lines. Dotted lines: guides to the eye. (d) Transmittance of a longitudinal wave incident with wave vector $\mathbf{k}_{\|}^{\bar{M}}$ on the slab of (c).

other, giving rise to a significant repulsion in the frequency domain. As the slab becomes thicker, the interaction between them is weaker and their repulsion smaller; therefore they come closer and closer. A typical example is given in Fig. 5(b), at the $\bar{M}$ point. This effect could be considered as evidence that these modes are not propagating along the $z$ direction, normally to the slab, but are strongly localized close to its surfaces. We have used the term surface-localized modes so far, without any direct proof, to describe them, because since these modes appear within the absolute gap region one expects that they will be attenuated inside the relatively thick slab. We can say that the phononic-crystal slab under consideration behaves, for frequencies within the gap, as a homogeneous and isotropic effective medium characterized by an imaginary wave number $\operatorname{Im}\left[k_{z}(\omega)\right]$ which determines the attenuation of the wave field over this region. ${ }^{26}$ As an example we consider the case described in Fig. 3(c), i.e., for $d_{s}=0.508 a_{0}$. For given $\omega$ and $\mathbf{k}_{\|}$ $=\left(2 \pi / a_{0}\right)(1 / 2, \sqrt{3} / 6)=\mathbf{k}_{\|}^{\bar{M}}$ (i.e., the wave vector parallel to the surface is at the $\bar{M}$ point of the SBZ), one can obtain this effective attenuation indirectly by the relation $\ln T=$ $-2 \operatorname{Im}\left(k_{z}\right) d N_{L}+$ const, where $T$ is the transmittance through a very thick slab $\left(N_{L} \gg 1\right)$. In this manner we find $\alpha$ $\equiv \operatorname{Im}\left(k_{z}\right) d=1.56$ for the resonance frequency $\omega a_{0} / c_{\text {air }}$ $=22.011$. The value of $\alpha$ coincides perfectly with that corresponding to the minimum imaginary part of $k_{z}$ along this crystallographic direction, as calculated at the given frequency from the complex band structure diagram of the infinite crystal. ${ }^{26}$ In order to verify the above hand-waving arguments, we also calculated the normalized field $|\mathbf{u}(\mathbf{r})|^{2} /\left|u_{0}\right|^{2}\left(\left|u_{0}\right|\right.$ is the amplitude of the incident wave) in the space between the different layers (interfaces or planes of spheres) of the slab when a longitudinal wave of frequency $\omega$ is incident on it with wave vector $\mathbf{k}_{\|}^{\bar{M}}$ (i.e., it propagates along the $\overline{\Gamma M}$ direction). The calculation was performed at points $\mathbf{r}_{i}, i=1,2, \ldots, N_{L}+1$, lying on the line passing through the centers of the unit cells of consecutive 2D (111) lattices, in the middle of the interlayer space. This is demonstrated in Fig. 5(c) for the resonance frequency $\omega a_{0} / c_{\text {air }}=22.011$ within the gap shown in Fig. 3(c), and for an off-resonance frequency $\omega a_{0} / c_{\text {air }}=21.00$ [no modes are observed at this frequency at the $\bar{M}$ point in the corresponding dispersion plot of Fig. 3(c)]. In the latter case (off resonance) the values of the field at these points $\mathbf{r}_{i}$ follow, apart from their oscillatory character, an exponential decay as can be seen from the dotted lines (guides to the eye) in Fig. 5(c). At the resonance again an exponential decay can be recognized, but now localization effects close to the surfaces are also present (see, for example, the relative enhancement of the field at the right interface). In both cases this exponential decay is described by $\ln \left[|\mathbf{u}(\mathbf{r})|^{2} /\left|u_{0}\right|^{2}\right]=-2 \tilde{\alpha} z / d+$ const, giving $\tilde{\alpha}=2.12$ at the resonance, and $\tilde{\alpha}=1.82$ for the off-resonance case. These values of $\tilde{\alpha}$ are close to those of $\alpha=1.56$ and 1.67, respectively, obtained from the transmittance for very thick slabs. Of course, one cannot expect better agreement between these two cases, since in the case of $\tilde{\alpha}\left(N_{L}=8\right)$ the thickness of the slab is relatively small and finite-size effects associated with the two surfaces dominate: in other words, the field close to the surfaces (within a depth of $N_{L} \sim 6$ layers for the present case) exhibits an oscillatory character which disappears for $z / d \gtrsim 6$, revealing a clear exponential decay, for this region of $z$. We checked this for a slab similar to those of Fig. 5(c) but consisting of $N_{L}=30$ layers, and a perfect agreement is found between $\alpha$ and $\tilde{\alpha}$, i.e., 1.56 at the resonance and 1.67 for the off-resonance case.

These surface modes manifest themselves as resonances in the transmission spectrum of a longitudinal wave incident on the slab. However, in contrast to resonances originating from propagating states of the finite slab, their height decreases on increasing the thickness of the slab. For relatively thin slabs, the two modes are coupled, giving rise to two distinct strong resonance peaks in the transmission spectrum. As the slab becomes thicker, these two peaks approach each other (the coupling is weaker) until they merge to a common resonance peak. After this point, the degree of coupling diminishes dramatically as we add more layers, and the height of the resonance decreases rapidly. A typical case is shown in Fig. 5(d) for a longitudinal wave incident with wave vector $\mathbf{k}_{\|}^{\bar{M}}$ on the slab described in Fig. 3(c), from the left. Of course the degree of coupling depends on several parameters (elastic or geometrical) of the system. For example, in a slab of a 


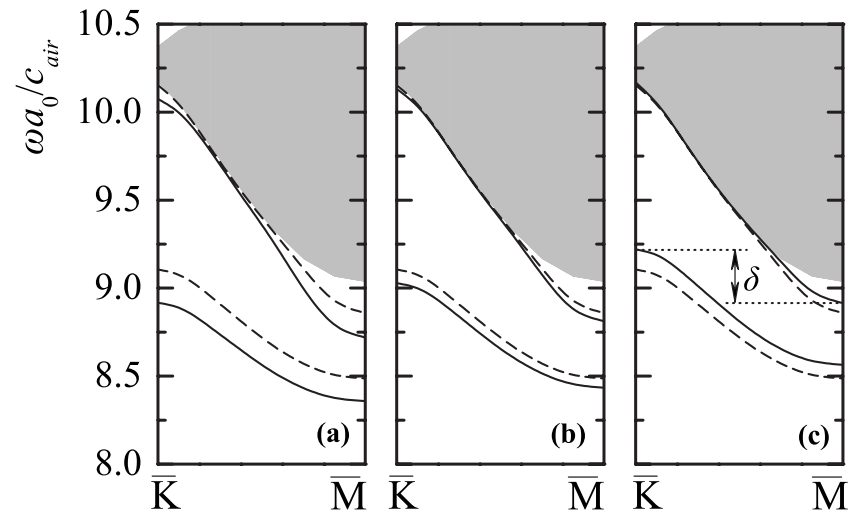

FIG. 6. Dispersion curves along the $\overline{K M}$ direction in the region below the lowest bulk bands of the infinite fcc crystal, for a slab consisting of eight fcc (111) layers of steel spheres of radius $S$ $=0.325 a_{0}$ in a polyester matrix, embedded in air. The two surfaces have a distance $d_{s}$ from the center of the surface planes of spheres $\left[d_{s}=\right.$ (a) $0.325 a_{0}=S$, (b) $0.375 a_{0}$, and (c) $0.458 a_{0}$ ]. Dashed lines: reference system $\left(d_{s}=0.408 a_{0}=d / 2\right)$, shown in Fig. 2 .

given number of layers of spheres, $N_{L}$, smaller $d_{s}$ values produce a stronger coupling, i.e., the repulsion between the two distinct peaks is stronger, and the fall in height of the double resonance peak observed in the transmission spectrum occurs at larger $N_{L}$ values. On the other hand, the presence of the second air-polyester interface is responsible for this splitting (the double resonance peak in the transmission spectrum). If it is removed, the second surface of the system is a virtual interface separating the phononic-crystal slab from the semi-infinite polyester at its right. The scattering on this interface is no longer so strong and it produces a resonance in a different frequency range; therefore, now only one resonance peak is observed in the corresponding transmission spectrum, whose height is (for the case presented here) about four orders of magnitude smaller than those presented in Fig. 5(d).

\section{The region below the first passband}

One can follow a similar analysis in order to investigate the behavior of the surface modes at the frequency region below the lowest bulk bands. We limit our study along the $\overline{K M}$ direction, but the same is valid for the rest of the highsymmetry lines of the SBZ. In Fig. 6 we show how the first surface branch, already shown in Fig. 2, is shifted along the frequency scale with a change in the distance of the airpolyester surfaces from the center of the surface layers of spheres, $d_{s}$. When the two surfaces are placed just at the position where the surface layers of spheres terminate $\left(d_{s}\right.$ $=0.325 a_{0}=S$ ), the branch is lowered in frequency with respect to the reference system [Fig. 6(a)], and the same is true for $S<d_{s}<d / 2$ [Fig. 6(b)]. When $d_{s}$ is increased more [Fig. $6(\mathrm{c})]$, this branch continues to go up toward the propagation modes of the slab (shaded region) until its penetration begins to take place (first at the $\bar{M}$ point, for $d_{s} \approx a_{0}$ ). However, the change in $d_{s}$ does not produce dramatic changes as in the case of the absolute band gap region. The surface-localized modes are less sensitive to such changes and, moreover, the
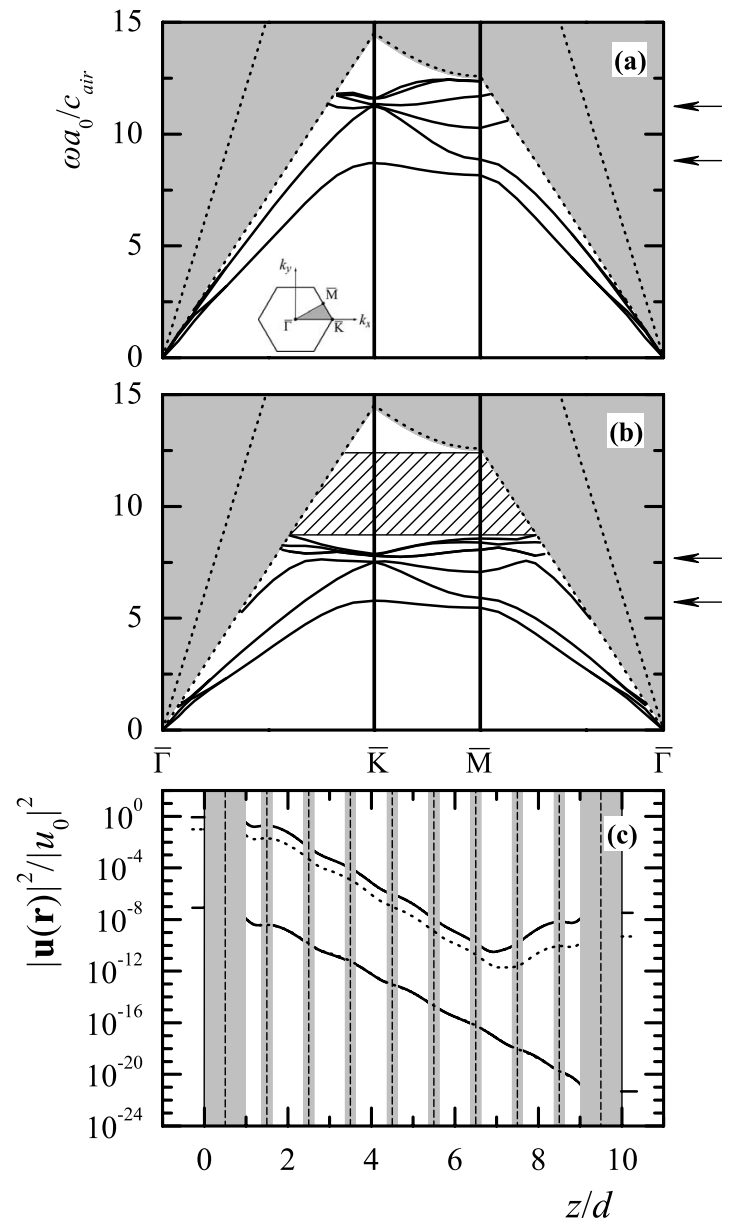

FIG. 7. Dispersion curves for a slab consisting of eight fcc (111) planes of steel spheres of radius $S=0.10 a_{0}$ in a polyester host, embedded in air, on both sides of which one additional surface layer of different sphere radius $S_{s}=0.40 a_{0}$ made of (a) steel or (b) tungsten is added between the air-polyester interface and the eight-layerthick phononic-crystal slab. Dotted lines: lines of propagation of transverse (lower branches) and longitudinal (upper branches) waves in infinite polyester. The arrows indicate the position of small gaps and the hatched region a large gap. (c) Same as Fig. 5(c) at the $\bar{M}$ point, for the system of (a) (solid lines) and for the corresponding system with the eight internal fcc (111) planes of steel spheres of radius $S=0.10 a_{0}$ replaced by a homogeneous polyester slab of the same thickness (dotted lines) at the resonance frequency $\omega a_{0} / c_{\text {air }}=8.165$ and 8.159 , respectively, (upper curves) and at offresonance frequency $\omega a_{0} / c_{\text {air }}=9.5$ (bottom curve). The small horizontal lines in the margins denote the values of the field intensity just before the air-polyester interfaces, in the region of polyester.

trend here is the opposite to that observed in the region of the absolute frequency gap: the higher the $d_{s}$, the closer the approach to the lower limit of the propagating-mode region. For that there exists a simple explanation: as $d_{s}$ increases we approach the case of a homogeneous polyester whose propagation lines are given in Fig. 2 as dotted lines. One could hope to create a small gap along the $\overline{K M}$ direction, between the first and the second surface-localized bands (solid lines in Fig. 6). For the present configuration this is not possible: the lower value of the second band (at the $\bar{M}$ point) is always 
below the higher value of the first band (at the $\bar{K}$ point). This distance $\delta$ (in units of $\omega a_{0} / c_{\text {air }}$ ) takes its lower value 0.198 for the case where the surfaces touch on the surface planes of spheres [Fig. 6(a)] and increases as we increase $d_{s}[\delta$ $=0.213$ and 0.301 for the cases of Figs. 6(b) and 6(c), respectively].

Although the change in $d_{s}$ does not offer possibilities for tuning such surface-localized modes at low-frequency regions, the case of varying $S_{s}$ does. In order to reveal these surface modes in full detail, one needs to use phononiccrystal slabs made of smaller spheres. In this case, the propagating modes are pushed up to higher frequencies, since the effective medium velocities of the phononic crystal tend to the values of the host matrix (polyester). On the other hand, the surface-localized modes appear at frequencies which are mainly determined by the value of $S_{s}$. An example is given in Fig. 7(a). The slab is now made of spheres of radius $S$ $=0.10 a_{0}$ resulting in a dispersion plot for the propagating modes (shaded region) almost identical to the propagation lines in the homogeneous polyester (dotted lines). In other words, the effective medium velocities of such a crystal are almost identical to those of the polyester host. Moreover, between the eight-layer-thick slab and the two air-polyester interfaces, we add now two surface layers of steel spheres of larger radius $S_{s}=0.40 a_{0}$ (keeping of course the same 2D periodicity), thus obtaining surface-localized modes quite low in frequency with respect to the propagating modes (compared to the case shown in Fig. 2), as can be seen in the corresponding dispersion diagram [Fig. 7(a)] as the solid lines. One can easily recognize in the modes appearing within the gap the general form of the dispersion diagram of a monolayer of spheres [see Fig. 5(a)]. A band folding is clearly seen around $\omega a_{0} / c_{\text {air }} \approx 11.3$ at the $\bar{K}$ and $\bar{M}$ points and the bottom of the hybridization gap is also revealed at about $\omega a_{0} / c_{\text {air }} \approx 12.5$. We note the presence of two very narrow gaps along the $K M$ direction extending for $\omega a_{0} / c_{\text {air }}$ from 8.708 to 8.863 and from 11.230 to 11.286 . It is worth noting that practically the same dispersion curves are obtained even if the eight internal layers of spheres of radius $S=0.10 a_{0}$ are removed and replaced by a homogeneous polyester slab of the same thickness. This could be of practical importance from the point of view of fabrication: a surface layer of spheres is enough to produce the effect.

When the steel spheres of the surface planes are replaced with tungsten ones (mass density $\rho_{\mathrm{s}}=19400 \mathrm{~kg} / \mathrm{m}^{3}$, longitudinal and transverse velocities $c_{l \mathrm{~s}}=5200 \mathrm{~m} / \mathrm{s}$ and $c_{t \mathrm{~s}}$ $=2900 \mathrm{~m} / \mathrm{s}$ ) the surface-localized modes are pushed down even more [Fig. 7(b)], an effect which is due mostly to the mass density difference between steel and tungsten. Now, apart from two small gaps (for $\omega a_{0} / c_{\text {air }}$ from 5.783 to 5.915 and from 7.636 to 7.751), a large region free of modes appears, above the surface-localized modes, extending from $\omega a_{0} / c_{\text {air }}=8.72$ to 12.43 .
Following the same procedure as that described in the discussion on Fig. 5(c), one can calculate the normalized field intensity in the interlayer space for the system of Fig. 7 (a) and for the corresponding system with the eight internal layers of spheres replaced by the homogeneous polyester. This is shown, for the sake of completeness, in Fig. 7(c) at the resonance frequencies of the two systems at the $\bar{M}$ point $\left(\omega a_{0} / c_{\text {air }}=8.165\right.$ and 8.159 , respectively $)$ and at an offresonance frequency $\left(\omega a_{0} / c_{\text {air }}=9.5\right)$. Similar conclusions to those made in the case of the absolute frequency gap region [Fig. 5(c)] are obtained, and now the oscillatory character of the field can be better distinguished. And, again, the same stands for the transmission coefficient: an analog to the behavior of Fig. 5(d) is also observed.

\section{CONCLUSIONS}

In this work, we presented results concerning the appearance of surface-localized modes inside the frequency gap regions of relatively thick solid 3D phononic-crystal slabs made of metallic spheres in a polyester matrix, embedded in air. We demonstrated that these modes can be tunable under an appropriate choice of the geometric or elastic parameters of the slab, giving rise to regions where no propagating and surface modes exist. Our findings could be useful in the construction and design of devices related to frequency filtering, waveguiding, etc. We considered here the case of finite slabs embedded in a symmetric environment, i.e., with the same semi-infinite medium (air) on both sides; however, the method can also be applied in an asymmetric system (for example, a plate placed on a solid substrate) without any problem. The study of plates supported on substrates should be the next step as it relates to a large category of practical applications. Moreover, the creation of linear or point defects on the surfaces of such systems (by removing some spheres or by changing the size and/or the material of some of them) should open another issue in this rapidly increasing subfield of phononic crystals. In this manner one could enlarge the possibilities offered by the 3D phononic-crystal plates by introducing tunable narrow defect bands within the large gaps found in the low-frequency regions of these systems [see Fig. 7(b)].

\section{ACKNOWLEDGMENTS}

R.S. gratefully acknowledges the hospitality of the UFR de Physique, Université de Lille 1, France. This work was supported by Le Fond Europeen de Developpement Regional (FEDER), INTERREG III France-Wallonie-Flandre (PREMIO) and le Conseil Regional Nord-Pas de Calais. 
*Present address: Instituto de Óptica-CSIC, Serrano 121, 28006, Madrid, Spain.

${ }^{1}$ For a comprehensive list of references on phononic crystals, see the phononic crystal database at http://www.phys.uoa.gr/ phononics/PhononicDatabase.html

${ }^{2}$ Y. Tanaka and S. I. Tamura, Phys. Rev. B 58, 7958 (1998); 60, 13294 (1999).

${ }^{3}$ E. V. Tartakovskaya, Phys. Rev. B 62, 11225 (2000).

${ }^{4}$ B. Manzanares-Martínez and F. Ramos-Mendieta, Phys. Rev. B 68, 134303 (2003).

${ }^{5}$ T. T. Wu, Z. G. Huang, and S. Lin, Phys. Rev. B 69, 094301 (2004).

${ }^{6}$ V. Laude, M. Wilm, S. Benchabane, and A. Khelif, Phys. Rev. E 71, 036607 (2005).

${ }^{7}$ J.-C. Hsu and T.-T. Wu, Phys. Rev. B 74, 144303 (2006).

${ }^{8}$ A. Khelif, B. Aoubiza, S. Mohammadi, A. Adibi, and V. Laude, Phys. Rev. E 74, 046610 (2006).

${ }^{9}$ J. O. Vasseur, P. A. Deymier, B. Djafari-Rouhani, and Y. Pennec, in Proceedings of International Mechanical Engineering Congress and Exposition, Chicago, Illinois, 2006, (ASME, Chicago, 2006), p.13353; J. O. Vasseur, P. A. Deymier, B. DjafariRouhani, Y. Pennec, and A.-C. Hladky-Hennion, Phys. Rev. B 77, 085415 (2008).

${ }^{10}$ J. O. Vasseur, A.-C. Hladky-Hennion, B. Djafari-Rouhani, F. Duval, B. Dubus, Y. Pennec, and P. A. Deymier, J. Appl. Phys. 101, 114904 (2007).

${ }^{11}$ X. Y. Zhang, T. Jackson, E. Lafond, P. Deymier, and J. O. Vasseur, Appl. Phys. Lett. 88, 041911 (2006).

${ }^{12}$ B. Bonello, C. Charles, and F. Ganot, Appl. Phys. Lett. 90, 021909 (2007).
${ }^{13}$ M. Torres, F. R. Montero de Espinosa, D. García-Pablos, and N. García, Phys. Rev. Lett. 82, 3054 (1999).

${ }^{14}$ F. Meseguer, M. Holgado, D. Caballero, N. Benaches, J. SánchezDehesa, C. López, and J. Llinares, Phys. Rev. B 59, 12169 (1999).

${ }^{15}$ R. E. Vines and J. P. Wolfe, Z. Kristallogr. 220, 810 (2005).

${ }^{16}$ S. Benchabane, A. Khelif, J.Y. Rauch, L. Robert, and V. Laude, Phys. Rev. E 73, 065601(R) (2006).

${ }^{17}$ R. Sainidou and N. Stefanou, Phys. Rev. B 73, 184301 (2006).

${ }^{18}$ K.-Y. Hashimoto, Surface Acoustic Wave Devices in Telecommunications: Modelling and Simulation (Springer, Berlin, 2000).

${ }^{19}$ O. Holmgren, J. V. Knuuttila, T. Makkonen, K. Kokkonen, V. P. Plessky, W. Steichen, M. Solal, and M. M. Salomaa, Appl. Phys. Lett. 86, 024101 (2005).

${ }^{20}$ T. T. Wu, L. C. Wu, and Z. G. Huang, J. Appl. Phys. 97, 094916 (2005).

${ }^{21}$ I. E. Psarobas, N. Stefanou, and A. Modinos, Phys. Rev. B 62, 278 (2000)

${ }^{22}$ R. Sainidou, N. Stefanou, I. E. Psarobas, and A. Modinos, Comput. Phys. Commun. 166, 197 (2005).

${ }^{23}$ R. Sainidou, N. Stefanou, and A. Modinos, Phys. Rev. B 69, 064301 (2004).

${ }^{24}$ R. Sainidou, N. Stefanou, and A. Modinos, Phys. Rev. B 66, 212301 (2002).

${ }^{25}$ B. A. Auld, Acoustic Fields and Waves in Solids (Wiley, New York, 1973), Vol. II.

${ }^{26}$ R. Sainidou, N. Stefanou, I. E. Psarobas, and A. Modinos, Z. Kristallogr. 220, 848 (2005). 